\title{
Dynamic Analysis for Derrick of Quadruple Stand Ultra-Deep Well Drilling Rig
}

\author{
WU Changliang", ZHOU Zhixiong, FANG Tai'an, ZUO Weidong
}

CNPC Engineering Technology R\&D Company Limited, Beijing 102206, China

National Engineering Laboratory of Oil and Gas Drilling Technology, Beijing 102206, China

*Corresponding Author: WU Changliang, CNPC Engineering Technology R\&D Company Limited, Beijing 102206, China

\begin{abstract}
Complicated formation and well depth over $7000 \mathrm{~m}$ in the piedmont region of Tarim Oilfield, China, lead to frequent tripping and more downhole accidents, and all of them cause the well construction period and costs increased significantly. $9000 \mathrm{~m}$ quadruple stand ultra-deep well drilling rig was developed to solve the above problems. The quadruple stand rig derrick was firstly modeled with $3 D$ design software, and then ANSYS was applied to the dynamic analysis of the derrick, including modal analysis, harmonic response and transient response. The analysis results show that the displacement in the front derrick opening direction of the crane, racking board and auxiliary racking board position is greater than the displacement in the direction of the derrick on both sides, and the low frequency forward derrick deformation should be pay more attention. The little displacement of the A-frame means that derrick will not produce large forced vibration in the process of drilling. The response displacement of the racking board is the largest and the response time is the longest under the action of transient impact load, so it is necessary to strengthen its support. The dynamic analysis provides the basis for the optimization design, strength checking and safety assessment of the derrick of quadruple stand rig.
\end{abstract}

Keywords: Quadruple Stand Ultra-Deep Well Drilling Rig Dynamics Analysis of Derrick Harmonic Response Analysis Transient Analysis

\section{INTRODUCTION}

To solve the problems of low drilling efficiency in Tarim piedmont region, the first 9000m quadruple stand drilling rig with independent intellectual property rights has been developed [1]. Quadruple stand drilling rigs use the quadruple stands instead of the convention triple ones, an auxiliary racking board is installed and the height of derrick and racking board are increased at the same time. The net height of the derrick increases from $48 \mathrm{~m}$ to $57.5 \mathrm{~m}$. The super high derrick will lead to the increase of load on the derrick, which cannot meet the requirements of strength and stiffness. Whether it is drilling or sticking accident, the vibration of derrick under the maximum static load is very dangerous. These vibrations will cause damage to drilling platform equipment and downhole tools, affect drilling speed, shorten the service life of drilling rigs, and reduce the safety of drilling operations. In addition, the derrick will be subjected to impact loads such as "quick lifting" and "sudden braking" in the process of tripping and drilling, while the static calculation cannot analyze the impact of these dynamic loads [2]. Therefore, it is necessary to carry out dynamic analysis of the derrick of quadruple stand drilling rigs, and study the variation of derrick structure response with frequency and transient impact load. The dynamic response of derrick can be determined.

\section{SOLID MODEL AND MESHING OF DERRICK}

Firstly, the solid model is built by 3D design software, and then the model is imported into ANSYS Workbench for finite element analysis [3]. In modeling, the following assumptions are made: the welding of each member is reliable and rigid connection; the derrick guardrail and ladder with little influence on the overall rigidity of derrick were neglected; the connection of each section of derrick is reliable and no relative displacement. The solid model and meshing of the derrick are shown in Figure 1. 


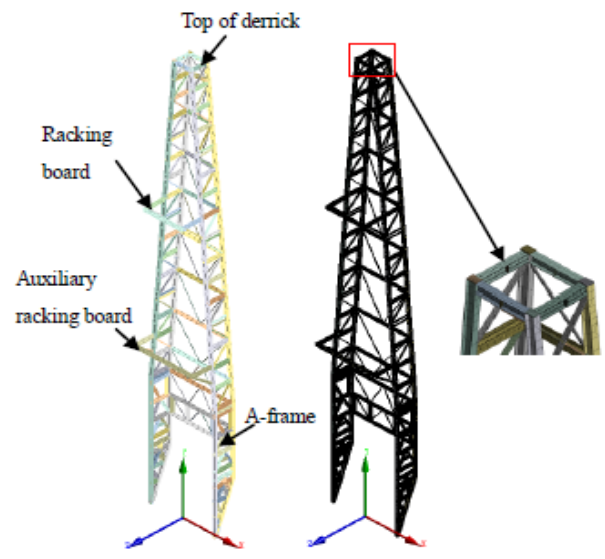

Figure1. Structural and meshing model of the derrick

\section{MODAL ANALYSIS OF DERRICK}

Modal analysis, the basis of dynamic analysis, is carried out before the analysis of harmonic response and transient response of derrick, because the natural frequencies and corresponding vibration forms of derrick can be obtained [2]. ANSYS software is used for derrick modal analysis, and Jacobian conjugate gradient solver is used by default. The first six modes of derrick are shown in Figure 2.

The results of modal analysis show that the derrick of quadruple stand drilling rig belongs to low frequency vibration system. Table 1 shows the results of the first six natural frequencies and modes of derrick. From the six modes, it can be seen that the maximum deformation of derrick structure is mainly located at the top of derrick and the racking board.
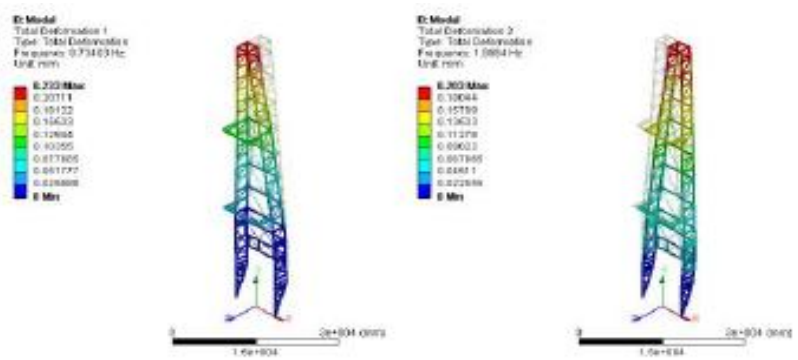

First-order mode

Second-order mode
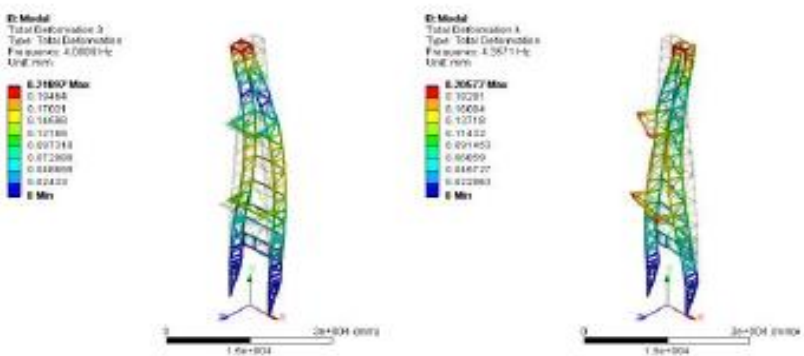

Third-order mode

Forth-order mode
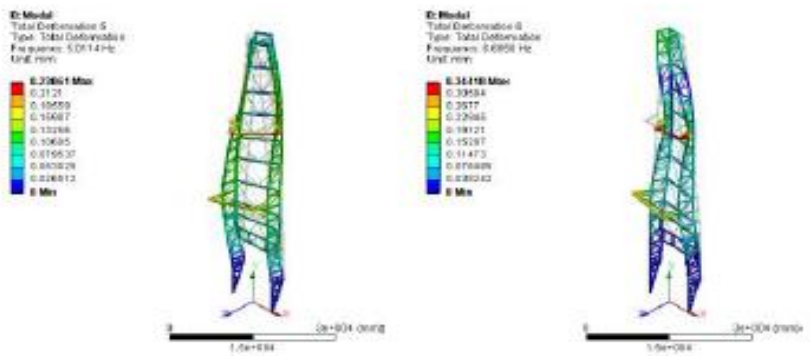

Fifth-order mode

Sixth-order mode

Figure2. First 6 modes of vibration pattern of the derrick 
Table1. Natural frequency and vibration mode results of the first 6 modes of the derrick

\begin{tabular}{|c|c|c|}
\hline Order & Natural frequency $(\mathrm{Hz})$ & Mode Characteristics \\
\hline 1 & 0.73 & Forward swing \\
\hline 2 & 1.99 & Backward swing \\
\hline 3 & 4.01 & Bending of upper derrick \\
\hline 4 & 4.35 & Torsion of upper derrick \\
\hline 5 & 5.01 & Torsion on both sides of derrick \\
\hline 6 & 6.68 & Global torsion \\
\hline
\end{tabular}

\section{HARMONIC RESPONSE ANALYSIS OF DERRICK}

The periodic load loaded on the structure will produce periodic response, harmonic response [4]. After the harmonic response analysis, the steady-state response of the linear structure under the load varying with time according to sinusoidal law can be obtained, the response of the structure at several frequencies and the response displacement curve and the frequency can be gotten, and then the peak response and the stress corresponding to the peak frequency can be found out [5].

\subsection{Theoretical Basis of Harmonic Response Analysis}

The differential equation of forced vibration of structures under harmonic loads [6].

$$
[M]\{\ddot{y}\}+[C]\{\dot{y}\}+[K]\{y\}=\{F\} \sin (\omega \cdot t)
$$

Where, $[M]$ is the mass matrix for structures; $[C]$ is the damping matrix for structures; $[K]$ is the stiffness matrix for structure; $\{\ddot{y}\}$ is the acceleration vectors; $\{\dot{y}\}$ is the velocity vector; $\{y\}$ is the displacement vector, $\{F\}$ is the amplitude vector of the exciting force on the structure; $\omega$ is the load frequency.

The node displacement response is that,

$$
\{y\}=\{A\} \sin (\omega t+\phi)
$$

Where, $\{A\}$ related to natural frequency, load frequency and damping matrix is the displacement amplitude vector. $\Phi$ is the phase angle of displacement response hysteretic excitation load.

The following formula can be obtained by substituting Formula (2) into Formula (1),

$$
H(\omega)=\frac{1}{1-\left(\frac{\omega}{\omega_{n}}\right)^{2}+2 i \varepsilon \frac{\omega}{\omega_{n}}}
$$

Where, $\omega_{n}$ is the natural frequency of the system; $\varepsilon$ is the system damping; $H(\omega)$ is the frequency response function.

\subsection{Harmonic Response Analysis of Derrick of Quadruple Stand Drilling Rig}

After loading $400 \mathrm{~N}$ on four surfaces on the top of derrick on average, the harmonic response of derrick of quadruple stand drilling rig is analyzed. The harmonic loading is shown in Figure 3.

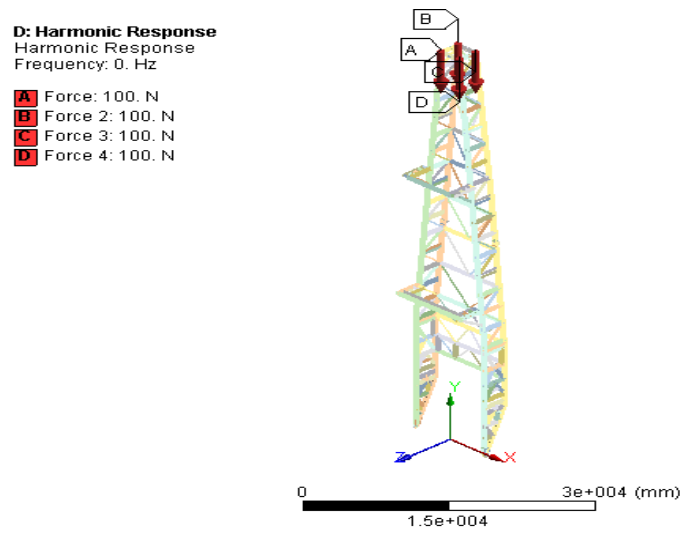

Figure3. Harmonic loading for the derrick 
In harmonic response analysis, the positions of top of derrick, racking board, auxiliary racking board and A-frame are taken to investigate the relationship between their displacements and frequencies. Figure 4 shows the displacement-frequency response curves of the top of derrick, racking board, auxiliary racking board and A-frame in X direction (both sides of derrick), Y direction (derrick height direction) and $\mathrm{Z}$ direction (front opening direction of derrick), respectively.

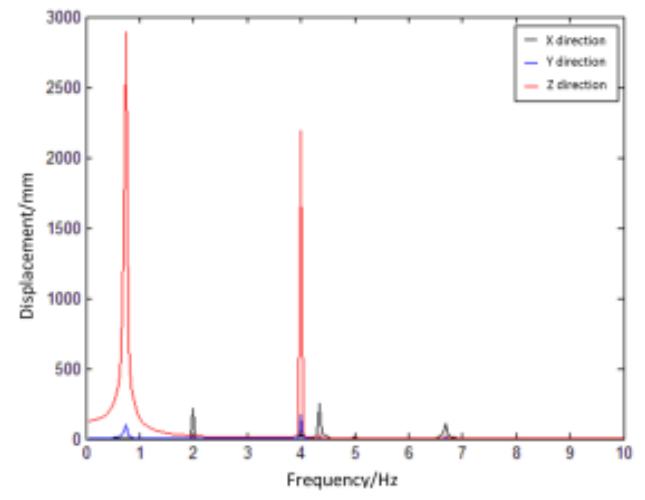

a. Top of derrick

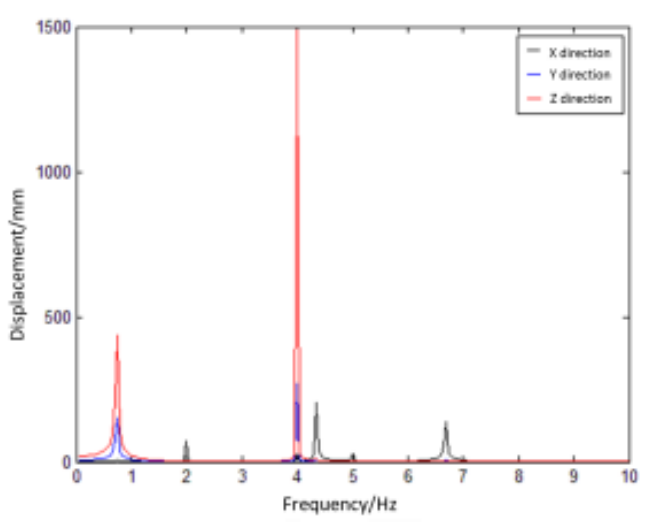

c. Auxiliary racking board

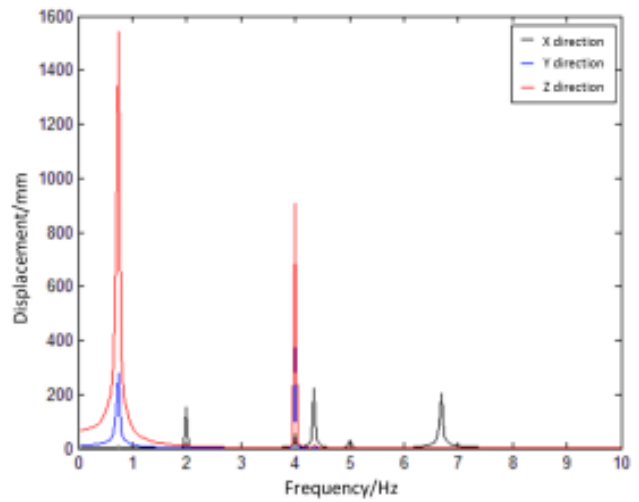

b. Racking board

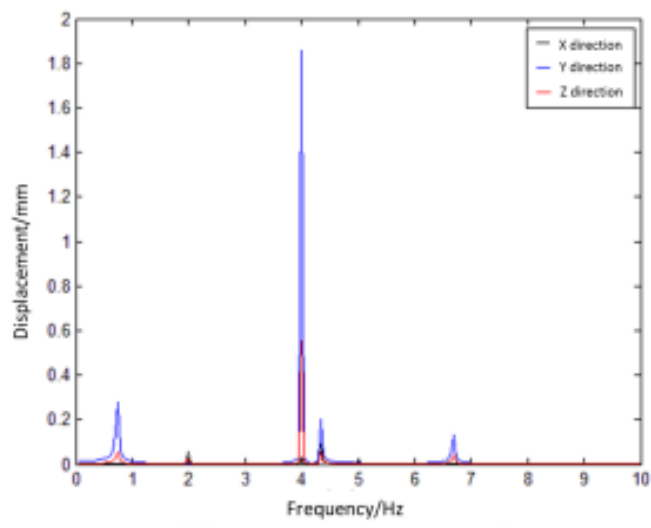

d. A-frame

Figure4. Displacement frequency response curves of different parts of the derrick

Figure 5 shows the vibration displacement response of derrick under the excitation frequency of $0.73 \mathrm{~Hz}$ and $4.01 \mathrm{~Hz}$.

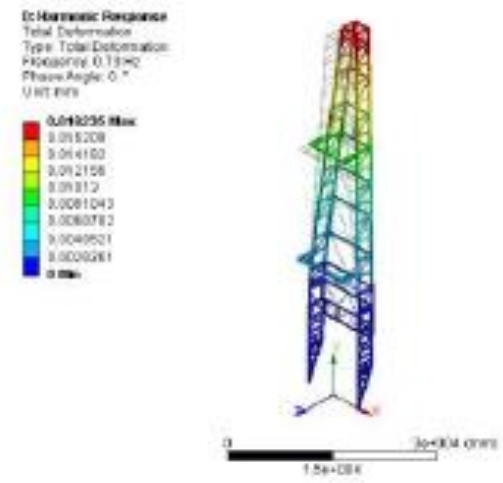

a. $0.73 \mathrm{~Hz}$

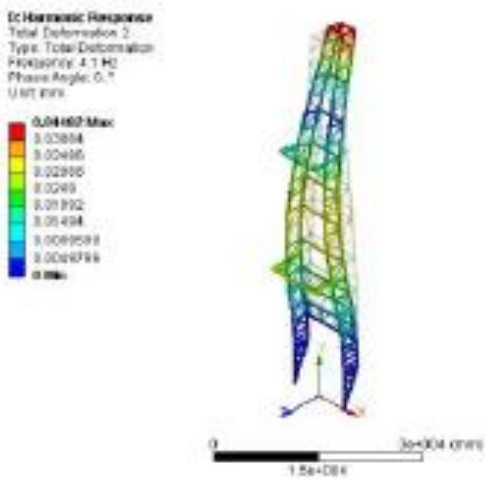

b. $4.00 \mathrm{~Hz}$

Figure5. Displacement response nephogram under $0.73 \mathrm{~Hz}$ and $4.00 \mathrm{~Hz}$ excitation frequency

From the results of Figure 4 and Figure 5, it can be see that:

(1) The results of harmonic response analysis are consistent with the results of modal analysis. The displacement responses of top of derrick, racking board, auxiliary racking board and A-frame near the natural frequencies of the derrick are peak values. The first-order natural frequencies $0.73 \mathrm{~Hz}$ and the 
third-order natural frequencies $4.01 \mathrm{~Hz}$ are dangerous excitation frequencies.

(2) The vibration law of the top of derrick and the racking board is similar, and the vibration is intense in the front opening direction. When the excitation frequency is close to the first natural frequency $0.73 \mathrm{~Hz}$, the displacement of the top of derrick and the racking board in the front opening are $2886 \mathrm{~mm}$ and $1542 \mathrm{~mm}$. When the excitation frequency is close to the third-order natural frequency $4.01 \mathrm{~Hz}$, the displacement of the top of derrick and the racking board in $\mathrm{Z}$ direction are $2192 \mathrm{~mm}$ and $905 \mathrm{~mm}$ respectively. Therefore, in the low frequency band of the derrick, attention should be paid to its forward deformation.

(3) Large vibration occurs at the position of the auxiliary racking board when excitation frequency is close to the third-order natural frequency of the derrick at $4.01 \mathrm{~Hz}$. The main vibration direction along the front open of the derrick and the displacement is $1487 \mathrm{~mm}$. large vibration and little displacement occur at the position of the A-frame when excitation frequency is the third-order natural frequency of the derrick $4.01 \mathrm{~Hz}$, which shows that there is no large forced vibration on A-frame when the derrick work.

\section{TRANSIENT RESPONSES ANALYSIS OF DERRICK}

Harmonic response analysis has some limitations, for example, it requires that all loads must change with time in accordance with sinusoidal law and have the same frequency, which can only reflect the linear characteristics of the structure. But the transient response analysis can solve the above problems [7]. Through the transient dynamic analysis, the displacement, strain and stress of the structure under the transient impact load can be obtained.

It is easy to produce large displacement and inertia force under the condition of "quick lifting" and "sudden braking" during tripping. Therefore, it is of great significance to study the deformation and strength of four key joints of derrick top, racking board, auxiliary racking board and A-frame under the action of huge transient impact load to understand the structural performance of derrick.

\subsection{Theoretical Basis of Transient Response Analysis}

Basic equation of motion for transient response of derrick structure [8 9],

$[M]\{\ddot{Q}(t)\}+[C]\{\dot{Q}(t)\}+[K]\{Q(t)\}=\{P(t)\}$

Where, $[M] 、[C] 、[K]$ are the mass, damping and stiffness matrices of derricks respectively; $\ddot{Q}(t) 、 \dot{Q}(t) 、 Q(t) 、\{P(t)\}$ are the acceleration vector, velocity vector, displacement vector and load vector of derrick model nodes respectively. The damping matrix $[C]$ can be calculated by multiplying Rayleigh damping constants alpha and beta by mass matrix and stiffness matrix [10].

$$
\begin{aligned}
& {[C]=\alpha[M]+\beta[K]} \\
& \left\{\begin{array}{l}
\alpha=\frac{2 \omega_{1} \omega_{2} \xi}{\omega_{1}+\omega_{2}}=\frac{4 \pi f_{1} f_{2} \xi}{f_{1}+f_{2}} \\
\beta=\frac{2 \xi}{\omega_{1}+\omega_{2}}=\frac{\xi}{\pi\left(f_{1}+f_{2}\right)}
\end{array}\right.
\end{aligned}
$$

Where, $\alpha$ is the mass damping, $\beta$ is the stiffness damping, $f_{1}$ is the first-order natural frequency of derrick and equals $0.73403 \mathrm{~Hz} ; f_{2}$ is the second-order natural frequency of derrick and equals $1.9984 \mathrm{~Hz}$. Substitute them in formula (6) and calculate, $\alpha$ equals $0.1349, \beta$ equals 0.00233 can be obtained. $\xi$ is the damping ratio of derrick. According to GB50011-2010 Code for Seismic Design of Buildings, derrick belongs to high-rise steel structure under wind load, and it is appropriate to take $\xi$ equal to 0.02 .

The transient response of derrick is analyzed by complete analysis method. Rayleigh damping is selected to calculate and loaded according to the time history of Figure 6. 


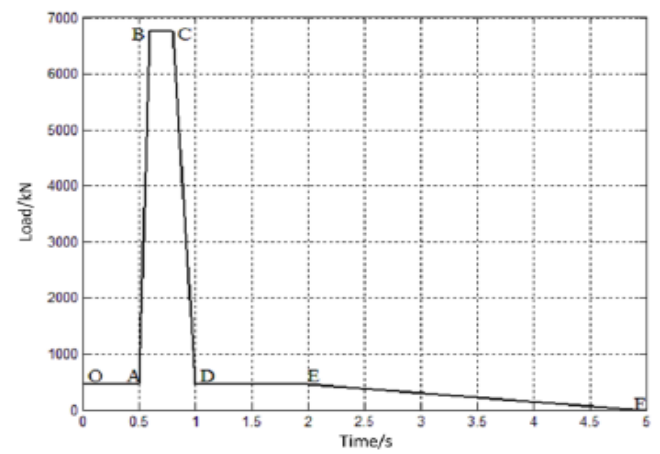

Figure6. Loading Time Course of Derrick

1) OA The top of derrick is subjected to a constant load of $464.85 \mathrm{kN}$.

2) $\mathrm{AB}$ When $\mathrm{t}=0.31 \mathrm{~s}$, the load on the top of derrick instantaneously increases to $6750 \mathrm{kN}$, and the whole process only takes $0.01 \mathrm{~s}$.

3) BC the top of derrick is subjected to the maximum hook load of $6750 \mathrm{kN}$ lasts for $0.008 \mathrm{~s}$.

4) $\mathrm{CD}$ The force on the top of derrick drops rapidly to $464.85 \mathrm{kN}$, which takes only $0.002 \mathrm{~s}$.

5) $\mathrm{DE}$ The top of derrick is affected by the gravity of swimming system $464.85 \mathrm{kN}$ lasts for $1.67 \mathrm{~s}$.

6) $\mathrm{EF}$ The load on the top of derrick decreases gradually until it drops to 0 at $5 \mathrm{~s}$.

\subsection{Analysis Results of Transient Response Of Derrick}

From figures 7,8 and 9, it can be seen that under the instantaneous impact, the A-frame is the most stable, while the racking board has the largest amplitude and the longest response time. The maximum displacement of the top of derrick is $18 \mathrm{~mm}$ in Z-axis direction at 0.72 seconds. The racking board has the largest corresponding transient load, the Z-axis direction is the most obvious, and the maximum value is $16 \mathrm{~mm}$, which appears at 0.64 seconds. The variation law of the auxiliary racking board is similar to that of the racking board. The A-frame is relatively stable under the transient load, and the rapidly attenuated displacement is mainly in Y-direction. The peak value of displacement response of derrick often appears in DE and EF sections, which indicates that the change of derrick displacement is slower than that of load.

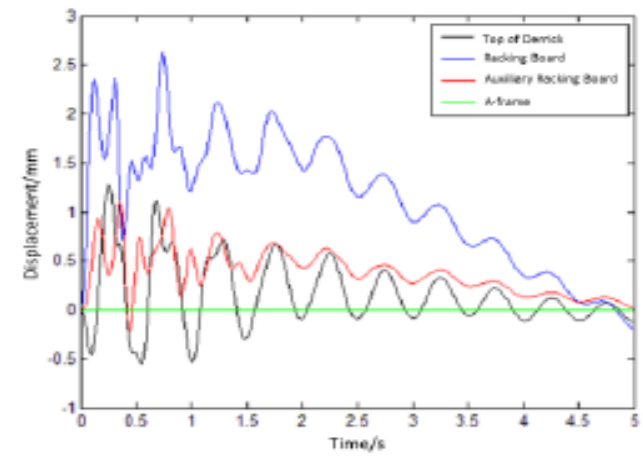

Figure7. Displacement-time curves of the four positions of derrick in X direction

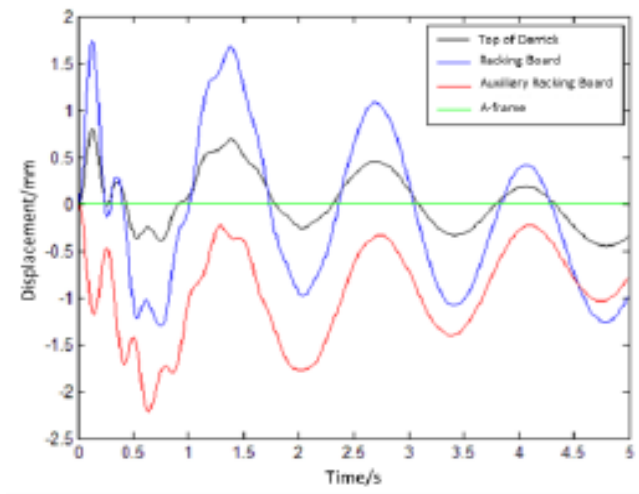

Figure8. Displacement-time curves of the four positions of derrick in Y direction 


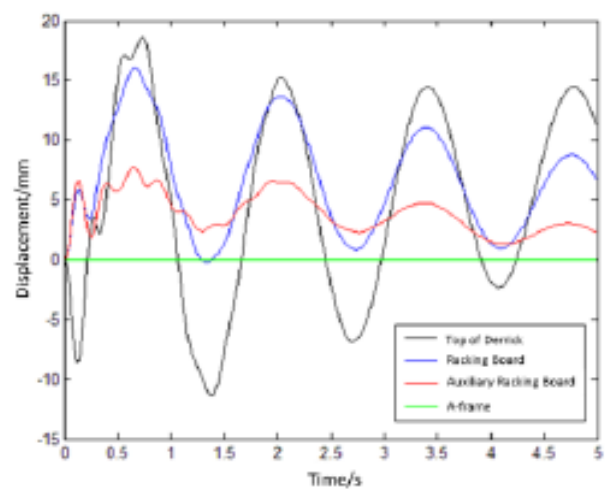

Figure9. Displacement-time curves of the four positions of derrick in $Z$ direction

\section{CONCLUSION}

(1) The front opening displacements of top of derrick, racking board and auxiliary racking board are larger than the lateral displacement of derrick, which indicates that the stiffness of the front opening direction of derrick is worse than that of both sides of derrick, and attention should be paid to the forward deformation of derrick in low frequency band. The displacement of each direction of the derrick position is very small, which indicates that the derrick will not produce large forced vibration when working on derrick.

(2) The comparison of harmonic response analysis and modal analysis shows that under the maximum static load, the dangerous excitation frequency approaches the first-order and third-order natural frequencies, which can cause the derrick resonance of the quadruple stand drilling rigs. It should be avoided the two dangerous excitation frequencies as far as possible in drilling operations.

(3) The derrick of quadruple stand drilling rigs has strong response under the cyclic loading. It can meet the derrick strength requirements by changing the higher strength level material, selecting reasonable material cross section and increasing the height of the A-frame.

(4) Under the action of transient impact load, the response displacement of the racking board is the largest and the response time is the longest, so the support needs to be strengthened.

(5) Field application in Tarim Oilfield shows that the derrick of quadruple stand drilling rig can effectively resist cyclic loading and transient impact, and it is safe and reliable.

\section{ACKNOWLEDGMENTS}

The authors would like to thank the China National Science and Technology Major Project "Automated Drilling Technology and Equipment for Deep and Ultra-deep Wells" for the grant and are also grateful to CNPC Tarim Oilfield Company, Bohai Drilling Engineering Company Ltd. and Baoji Oilfield Machinery Co., Ltd for the help in writing the paper.

\section{REFERENCES}

[1] Sun Tingwen, Yang Yu. The first $9000 \mathrm{~m}$ quadruple standpipe rig in China was opened in Xinjiang. Geological equipment, 2013 (4): 5-5

[2] Zou Longqing. Analysis for the dynamic response of drilling rig derricks. Harbin Engineering University, 2006

[3] Liu Yujuan, Ding Qingxin, Zhang Suian, et al. FEM analysis of derrick structure of SMJ5540TZJ15/800Y CBM drilling. Coal mine machinery, 2010, 31 (1): 97-99

[4] Zhou Sizhu, Zhu Keqiang, Hua Jian, et al. Harmonic response analysis of deep-well drilling rig derrick and substructure system. China Petroleum Machinery, 2013, 41 (8): 28-31

[5] Zou Chunyu, Ren Fang, Yang Zhaojian. Harmonic response analysis for main shaft device of single rope winding mine hoist based on ANSYS WORKBENCH. Coal Engineering, 2017, 49 (3): 100-102,106

[6] Liang Qinghai, Zhou Guoqiang, Han Dongying. The analysis of dynamics characteristic for JJ454/49-H type ocean dynamics derrick. Oil Field Equipment, 2007, 36(4):34-37

[7] Wang Kai,Zhang Shimin. Dynamic characteristics analysis of fracturing truck frame. Science Technology and Engineering, 2017,17(31):346-351

[8] Wang Kai,Yao Jin, Deng Xingqiao, et al. Transient dynamics analysis for the end face engagement worm gear. Advanced Engineering Sciences, 2017, 49(1): 86-94 
[9] He Minghui, Liu Decheng, Nie Jingxu. Study on transient dynamic response of thin conical shells. Vibration and shock, 2006, 25 (6): 157-159

[10] Wu Chang, Ai Zhijiu, Chen Hailin, et al. Analysis of dynamics characteristic for JJ250/42-K type drilling derrick. Oil Field Equipment, 2009,38(11):19-23

Citation: WU Changliang, et.al (2019). "Dynamic Analysis for Derrick of Quadruple Stand Ultra-Deep Well Drilling Rig”, International Journal of Petroleum and Petrochemical Engineering (IJPPE), 5(1), pp.30-37, DOI: http://dx.doi.org/10.20431/2454-7980.0501005

Copyright: (C) 2019 Authors. This is an open-access article distributed under the terms of the Creative Commons Attribution License, which permits unrestricted use, distribution, and reproduction in any medium, provided the original author and source are credited 